\title{
Structure based design of potent selective inhibitors of protein kinase D1 (PKD1)
}

\author{
Jianwen A. Feng ${ }^{\star}, \mathrm{a}, \dagger$, Patrick Lee ${ }^{a}$, Moulay Hicham Alaouia, Kathy Barretta, \\ Georgette Castanedo ${ }^{a}$, Robert Godemannc, Paul McEwan ${ }^{\mathrm{d}}$, Xiaolu Wang ${ }^{\mathrm{c}}$, Ping \\ Wu $^{a}$, Yamin Zhang ${ }^{b}$, Seth F. Harris ${ }^{a}$, Steven T. Staben*,a \\ aGenentech, 1 DNA way, South San Francisco, CA 94080 \\ bPharmaron Beijing Co., Ltd. 6 Taihe Road, BDA, Beijing, P.R. China, 100176 \\ ${ }^{c}$ Evotec AG, Manfred Eigen Campus, Essener Bogen, Hamburg, Germany 22419 \\ dEvotec (UK) Ltd, 114 Innovation Dr, Milton Park, Abingdon OX14 4Rz, UK
}

\section{Table of contents}

Experimental details for PKD1 Enzyme inhibition assay 2

Experimental details for structural biology (mNIK and Map4K4) 3

Kinase selectivity data for compounds 10,11 and (S)-12 4

Synthetic procedures for (R)-7, 10,11 and (S)-12 9

$\begin{array}{lr}\text { Modeling software and procedures } & 19\end{array}$ 


\section{PKD1 Enzyme Inhibition Assay}

The ability of the Protein kinase D1 (PKC $\mu$ or PRKD1) to catalyze the hydrolysis of adenosine-5'-triphosphate (ATP) was monitored using the Transcreener ADP (adenosine-5'-diphosphate) assay (BellBrook Labs). Purified PKD1 (1.5 nM) sourced from Life Technologies was incubated with test compounds for 1-2 hours in $50 \mathrm{mM}$ 2-Amino-2-hydroxymethyl-propane-1,3-diol buffer (pH 7.5) containing $10 \mathrm{mM}$ $\mathrm{MgCl}$, $2 \mathrm{mM}$ dithiothreitol, $10 \mathrm{mM}$ ATP, $0.01 \%$ Triton $\mathrm{X}-100,0.02 \%$ gammaglobulins from bovine blood, $1 \mathrm{mM}$ ethylene glycol-bis(2-aminoethylether)$\mathrm{N}, \mathrm{N}, \mathrm{N}^{\prime}, \mathrm{N}^{\prime}$-tetraacetic acid, 1\% dimethylsulfoxide (DMSO), $7 \mathrm{mg} / \mathrm{mL}$ ADP antibody and $5 \mathrm{nM}$ ADP-MR121 633 tracer. Reactions were quenched by the addition of 20 mM 2,2',2",2'"-(ethane-1,2-diyldinitrilo)tetraacetic acid and 0.01\% Brij 35. The tracer bound to the antibody was displaced by the ADP generated during the PKD1 reaction, which causes a decrease in fluorescence polarization that was measured by laser excitation at $633 \mathrm{~nm}$ with a Fluorescence Correlation Spectroscopy Plus reader (Evotec AG). Equilibrium dissociation constant $\left(K_{\mathrm{i}}\right)$ values for PKD1 inhibitors are calculated from plots of activity vs. inhibitor concentration using Morrison's quadratic equation that accounts for the potential of tight binding, and by also applying the conversion factor that accounted for competitive inhibition and the concentration of substrate used in the assay relative to its Michaelis constant $\left(K_{\mathrm{m}}\right)$. 


\section{Crystallography:}

Apo mNIK (329m-675m) protein solution was concentrated to $9.5 \mathrm{mg} / \mathrm{ml}$ in $20 \mathrm{mM}$ Tris-HCl (pH 8.0), $150 \mathrm{mM} \mathrm{NaCl}, 4 \%$ glycerol, and 1 mM TCEP (Buffer B) supplemented with $1 \mathrm{mM}$ of the CDK2 inhibitor NU6140 (Calbiochem). Crystals were grown at $4^{\circ} \mathrm{C}$ in hanging drops with a reservoir solution of $100 \mathrm{mM}$ sodium citrate tribasic dihydrate ( $\mathrm{pH}$ 6.2), $0.5 \mathrm{M}$ ammonium sulfate, $0.9 \mathrm{M}$ lithium sulfate and were cryoprotected in crystallization well solution plus 15\% xylitol (QIAGEN \#133292) and 15\% D-(+)-sucrose (QIAGEN no. 133191). Data were collected at Diamond Light Source beamline I04-1.

MAP4K4 protein purification, crystallization, and structure refinement were previously described in Crawford al. ${ }^{1}$

Portions of this research were carried out at the Stanford Synchrotron Radiation Lightsource, a Directorate of the SLAC National Accelerator Laboratory and an Office of Science User Facility operated for the U.S. Department of Energy Office of Science by Stanford University. The SSRL Structural Molecular Biology Program is supported by the DOE Office of Biological and Environmental Research, and by the National Institutes of Health, National Institute of General Medical Sciences and the National Center for Research Resources. The contents of this publication are solely the responsibility of the authors and do not necessarily represent the official views of NIGMS, NCRR, or NIH. The authors thank the staff at Diamond Light Source beamline I04-1 and Stanford Synchrotron Radiation Lightsource beamline 12-2. 
Kinase selectivity for compounds 10,11 and (S)-12.

Determined as a \% inhibition at a single, indicated test concentration at Invitrogen $®$.

\begin{tabular}{|c|c|c|c|}
\hline & Compound (S)-12 & Compound 10 & Compound 11 \\
\hline TEST CONCENTRATION & $0.1 \mu \mathrm{M}$ & $0.1 \mu \mathrm{M}$ & $0.1 \mu \mathrm{M}$ \\
\hline \multicolumn{4}{|l|}{ KINASE } \\
\hline ACVR1B & 5.9 & 1.9 & 2 \\
\hline ACVR2B & 3.3 & 12.6 & 2.3 \\
\hline AKT1 & 3.5 & 2.2 & 4.3 \\
\hline AKT2 & 6 & 0.5 & -5.8 \\
\hline ALK2 & 1.1 & 1.7 & 1.4 \\
\hline ARK5 & -1.6 & 15.5 & 1.7 \\
\hline ASK1 & 9.5 & 7.5 & 4.8 \\
\hline Abl & -3.8 & -2 & 1.9 \\
\hline Aurora_A & 8.1 & 5.5 & 2 \\
\hline Aurora_B & 13.5 & 44.6 & 41.7 \\
\hline Aurora_B(+1) & -3.5 & & 13.5 \\
\hline Axl & -4.5 & 4.1 & -8 \\
\hline BMPR1A & 14.9 & 1.6 & 7.1 \\
\hline BRAF & -0.5 & 2.4 & 2.3 \\
\hline BTK & -9.9 & -1.2 & -4.9 \\
\hline Blk & 0.9 & 8 & 2.4 \\
\hline Bmx & 7.9 & 7 & 5.6 \\
\hline BrSK1 & -0.1 & 2.8 & -6.4 \\
\hline Brk & -2.8 & -0.1 & -5.9 \\
\hline CAMKK1 & 0.8 & 0.5 & 9.2 \\
\hline САМКК2 & 3.3 & -1.6 & 0.6 \\
\hline CDK1/cyclinB & 8 & 5 & 5.6 \\
\hline CDK2/cyclinA & 6.7 & 7.6 & 5.2 \\
\hline CDK5/p25 & 4 & 5 & 5.3 \\
\hline CDK7/cyclinH & -10.1 & -10.1 & -3.3 \\
\hline CDK8/cyclinC & 3.1 & 3.9 & 3.3 \\
\hline CDK9/cyclinT1 & -8.8 & 1.1 & -1.4 \\
\hline CHK1 & 10.8 & 6.3 & 1.5 \\
\hline CHK2 & 6.3 & 8 & 3.1 \\
\hline CK1_alpha1 & 3.2 & 6 & 4 \\
\hline CK1_delta & 4.7 & 2.4 & -2.6 \\
\hline CK1_epsilon1 & 3.4 & -1.8 & -0.6 \\
\hline CK1_gamma1 & 7 & 5.6 & 17.2 \\
\hline CK1_gamma2 & 14.2 & 4.4 & 4.6 \\
\hline CK2_alpha1 & 2.4 & 10.2 & 7.3 \\
\hline CLK1 & 3.7 & 4.9 & 5.3 \\
\hline CLK2 & 5.2 & 0.6 & 3 \\
\hline CLK3 & 6.9 & 5.3 & 1.3 \\
\hline CLK4 & 1.6 & -3 & 2.4 \\
\hline CSF1R & -2.1 & 4.2 & -3.9 \\
\hline CSK & 9.9 & 15.8 & -1.8 \\
\hline CaMKI & 28.6 & 24.2 & 1.8 \\
\hline CaMKII_beta & -1 & -7.6 & -12.1 \\
\hline CaMKI_delta & -8.7 & 5.1 & -8 \\
\hline CamKII_alpha & 3.7 & 1.8 & 0.2 \\
\hline
\end{tabular}




\begin{tabular}{|c|c|c|c|}
\hline CamKIV & -8.9 & -13.2 & -11.4 \\
\hline $\operatorname{Cot}(+1)$ & -1 & & -3 \\
\hline DAPK1 & 2.9 & 4.5 & 9.3 \\
\hline DCAMKL2 & 8.1 & 8.5 & 3.7 \\
\hline DDR1 & 1.8 & 1.6 & 1 \\
\hline DMPK & 0.7 & 2.2 & 4 \\
\hline DNA-PK & 4.9 & 1.6 & 2.1 \\
\hline DRAK1 & 2.3 & 0.6 & 2.7 \\
\hline DYRK1A & 6.3 & 5.2 & 5.3 \\
\hline DYRK3 & -0.4 & -2.1 & 3 \\
\hline DYRK4 & 4.4 & -2 & 0.3 \\
\hline EGFR & -0.7 & 0 & 3.3 \\
\hline EGFR(T790M,L858R) & -1.3 & 3.5 & -2.9 \\
\hline ERK2 & 7.4 & 6 & 9.9 \\
\hline EphA1 & 2.6 & 2.2 & -8.2 \\
\hline EphA3 & 4 & 3.3 & 8.9 \\
\hline EphA7 & 7.2 & 2.9 & 8.6 \\
\hline EphA8 & -3.4 & -2.6 & -4.8 \\
\hline EphB1 & 2.3 & 4.2 & 2.5 \\
\hline EphB3 & 0.6 & 1.8 & 2.2 \\
\hline ErbB2 & -7.7 & -5 & 1.2 \\
\hline ErbB4 & 4.1 & 1.8 & 7.6 \\
\hline FAK & 12.4 & 9.4 & 10.4 \\
\hline FGFR1 & -1.8 & -10.3 & -3.6 \\
\hline FGFR3 & -4.2 & -0.1 & -0.7 \\
\hline FGFR4 & 4.4 & 3.7 & 4.3 \\
\hline Fes & 7.8 & 10.3 & -0.6 \\
\hline Fgr & 2.1 & 3 & -1 \\
\hline Flt1 & 2.1 & -0.9 & 2.2 \\
\hline Flt3 & 13.5 & 12.6 & 14 \\
\hline Flt4 & -2.5 & 20.7 & -5.4 \\
\hline Flt4(+1) & -7.8 & & 1.3 \\
\hline Frk & 1.4 & 0.3 & 6.1 \\
\hline GRK2 & 5.3 & 3.6 & 1.7 \\
\hline GRK3 & -6.4 & -3.3 & -6.9 \\
\hline GRK5 & 1.7 & -2.4 & -2.3 \\
\hline GRK6 & 7 & 5.4 & 9.4 \\
\hline GSK3_alpha & 6.8 & 6.8 & 6.9 \\
\hline GSK3_beta & -2 & 0.1 & 8.9 \\
\hline HIPK1 & 0.5 & 0.3 & -3.5 \\
\hline HIPK2 & -1.4 & 2.1 & -4.9 \\
\hline HIPK4 & 2.5 & 2 & 2.7 \\
\hline Hyl & 6.4 & -0.1 & -2.3 \\
\hline IGF1R & 5.4 & 2.1 & 3.3 \\
\hline IKK_alpha & 13.3 & 5.8 & -1 \\
\hline IKK_beta & 0.4 & -0.6 & 5.7 \\
\hline IKK_epsilon & 9.7 & 7.2 & 5.9 \\
\hline IRAK1 & 9.7 & 3.2 & 2.5 \\
\hline IRAK4 & 4.6 & 2.8 & 8 \\
\hline IRR & 7.4 & 4.6 & -0.2 \\
\hline ITK & -2.8 & 3 & 9.4 \\
\hline InsR & 1 & 3 & -1.3 \\
\hline JAK1 & 1.7 & -1.8 & 0.7 \\
\hline JAK2 & 1.7 & -0.6 & 2.6 \\
\hline
\end{tabular}




\begin{tabular}{|c|c|c|c|}
\hline JAK3 & -0.8 & -5 & -4.3 \\
\hline JNK1(+1) & 11.8 & & 14.2 \\
\hline JNK1_alpha1 & 11.6 & 13.5 & 13.2 \\
\hline JNK2 & 6.5 & 20 & 13.4 \\
\hline JNK3 & 5 & 9 & 10.6 \\
\hline KDR & 4.1 & 24.9 & 3.9 \\
\hline KDR(+1) & -0.4 & & 4.4 \\
\hline KHS1 & 6.9 & 8.8 & -0.7 \\
\hline KHS1(+1) & 6.6 & & 16.4 \\
\hline Kit & -3 & -3.2 & -4.3 \\
\hline LIMK1 & 2.3 & 6.7 & 7.3 \\
\hline LRRK2 & 0.7 & 35.7 & 0.5 \\
\hline LRRK2(+1) & -1.3 & & 8.2 \\
\hline LTK & 0.2 & 2.3 & 7.6 \\
\hline Lck & 8.9 & 6.8 & -2.9 \\
\hline Lyn & 3.5 & 4.1 & -6.4 \\
\hline MAP4K4 & 6.3 & 56.7 & 14.1 \\
\hline MAP4K4(+1) & 0 & & 20.5 \\
\hline МАРКАРК2 & 3 & 2.3 & 0.8 \\
\hline МАРКАРКЗ & -5.8 & -2.5 & 5.7 \\
\hline MARK1 & 5.4 & 5.1 & 7.6 \\
\hline MARK3 & 4.3 & 5.6 & 6.1 \\
\hline MEK1 & 6.6 & 20.3 & 20.2 \\
\hline MEK1(+1) & -1 & & -0.5 \\
\hline MEK3 & 4.2 & 20.5 & 18.3 \\
\hline MEKK2 & 2.3 & 11.3 & 24.9 \\
\hline MEKK2(+1) & 5.5 & & 26.5 \\
\hline MELK & 9.9 & 5.4 & -2.9 \\
\hline МКK6 & 5.1 & 35.2 & 25.9 \\
\hline MKK6(+1) & 0.5 & & 7 \\
\hline MKNK1 & 4.1 & 3.3 & 1.1 \\
\hline MKNK2 & 1.9 & 5.9 & 13.4 \\
\hline MLK1 & 2.7 & 16.2 & 8 \\
\hline MLK2 & 5.8 & 44.3 & 4.1 \\
\hline MLK2(+1) & 3.6 & & 4.4 \\
\hline MRCK_alpha & 5.4 & 9.9 & 7.3 \\
\hline MSK1 & 1.4 & 4.4 & -1.8 \\
\hline MSSK1 & 10.5 & 8.7 & 0.2 \\
\hline MST1 & 3.2 & 0.7 & 3.2 \\
\hline MST2 & -1.3 & 4.9 & -0.3 \\
\hline MST3 & 2.6 & 3.5 & 4 \\
\hline MST4 & 5.5 & 6.5 & 5.8 \\
\hline MYLK(smMLCK) & 0.4 & -0.2 & 2.1 \\
\hline MYLK3(caMLCK) & 4.7 & 5.6 & 7 \\
\hline Mer & 8.7 & 3.3 & -0.5 \\
\hline Met & 6.3 & 8.6 & 7.7 \\
\hline Mink1 & 6.8 & 54.2 & 20.3 \\
\hline Mink1(+1) & 2.2 & & 9.9 \\
\hline MuSK & -2 & 2.1 & -14.7 \\
\hline NEK1 & 18.5 & 8.5 & 0.3 \\
\hline NEK4 & 17.7 & 2.5 & -0.7 \\
\hline NEK6 & 0 & 2 & -3.3 \\
\hline NEK9 & -1.1 & 0.4 & -6.2 \\
\hline NLK & 5.1 & 5.1 & 3.4 \\
\hline
\end{tabular}




\begin{tabular}{|c|c|c|c|}
\hline PAK1 & 8.4 & 11.5 & 9.4 \\
\hline PAK3 & -12 & 7.6 & 10.9 \\
\hline PAK4 & 6.3 & 7.3 & 7.4 \\
\hline PAK4(+1) & -8.5 & & -5.5 \\
\hline PAK6 & 11.1 & 3.8 & -1.4 \\
\hline PASK & 1.9 & -2.8 & -3.8 \\
\hline PDGFR_alpha & 0.4 & 0.2 & 0.9 \\
\hline PDK1(direct) & 12.1 & -0.9 & 2.2 \\
\hline PI3K-A & 4.7 & 3.2 & -10.5 \\
\hline PI3K-G & 20.4 & 23.5 & 12 \\
\hline PI3K-G(+1) & -4.6 & & -6.3 \\
\hline PIM1 & -3.4 & -5.5 & 1.9 \\
\hline PKA & -4.8 & -3.5 & -2.5 \\
\hline PKC_alpha & 7.9 & 7.3 & 1.5 \\
\hline PKC_beta1 & 11 & 9.6 & 2.4 \\
\hline PKC_delta & 0.4 & -11.2 & -9.2 \\
\hline PKC_epsilon & 0.8 & 1 & -3.3 \\
\hline PKC_eta & 10.1 & 10.6 & 6.1 \\
\hline PKC_theta & 7.6 & 7.7 & 9.4 \\
\hline PKC_zeta & 6.7 & 8.8 & 8.9 \\
\hline PKD1 & 88.6 & 55.6 & 83.6 \\
\hline PKD1(+1) & 61.9 & & 90.3 \\
\hline PKG1_alpha & -4.7 & 0.4 & -1.7 \\
\hline PLK1 & 6.5 & 3.8 & 3 \\
\hline PLK2 & 7.9 & 4.9 & 5.7 \\
\hline PLK3 & -6.2 & 1.2 & 1.2 \\
\hline PRAK & 6.6 & 2.3 & -2.5 \\
\hline PRK1 & 7.7 & -3 & -5.5 \\
\hline PRKAA1 & 0.6 & 6.1 & 2.9 \\
\hline PhK_gamma1 & -11.7 & 1.8 & 0.9 \\
\hline PhK_gamma2 & 1.6 & 1.4 & 2.3 \\
\hline PrKX & 1.2 & 3.7 & -5.4 \\
\hline RAF1(Y340D,Y341D) & -3.1 & -3.7 & -1.1 \\
\hline RIPK2 & 2.1 & 2.5 & 1.1 \\
\hline ROCK1 & 6.2 & 5.2 & 4.4 \\
\hline ROCK2 & 6.5 & 16.5 & 14.6 \\
\hline RSK1 & 9.1 & 10.6 & 12.3 \\
\hline RSK2 & 2.3 & 3.6 & 4.8 \\
\hline RSK3 & 5.9 & 4.4 & 4.1 \\
\hline Ret & -9.3 & 14.2 & 6.2 \\
\hline Ron & 5 & 5.3 & -3.7 \\
\hline Ros & 3.4 & 2 & -8.2 \\
\hline Rse & 5.2 & 4 & -3.3 \\
\hline SGK1 & -6.6 & -5.2 & -5.2 \\
\hline SGK2 & 2.9 & 2.5 & 2.6 \\
\hline SGK3 & -3 & 0.6 & 1.2 \\
\hline SIK2 & 6.6 & 2.7 & -9.3 \\
\hline SLK & 1.3 & 1.6 & 0.9 \\
\hline SPHK1 & 12.8 & 12.8 & -7.4 \\
\hline SRPK1 & 1.2 & -2.2 & -13.6 \\
\hline STK16 & -0.2 & 0.1 & 3.3 \\
\hline STK33 & 1.7 & 5.9 & 1.1 \\
\hline Src & 23.2 & 18.7 & 3.4 \\
\hline $\operatorname{Src}(+1)$ & 2.5 & & 2.5 \\
\hline
\end{tabular}




\begin{tabular}{|c|c|c|c|}
\hline Srm & -4.3 & 0.2 & 2.5 \\
\hline Syk & 0.7 & -0.6 & -0.8 \\
\hline TAK1-TAB1 & 2.1 & 36.4 & 5.7 \\
\hline TAK1-TAB1(+1) & 7.4 & & 10 \\
\hline TAO2 & 7.9 & -0.8 & 4 \\
\hline TBK1 & 12.1 & 4.9 & 0.4 \\
\hline TEC & 4.5 & 4 & 5.6 \\
\hline TGFBR1 & -0.8 & 1.4 & 8.9 \\
\hline TNK2 & 2.2 & 3.5 & 5.5 \\
\hline TSSK1 & 3.1 & 1.2 & 2.7 \\
\hline TSSK1(+1) & 3.1 & & 4.3 \\
\hline TTK & 9 & 14.7 & 12.5 \\
\hline TXK & 17.4 & 13.7 & -0.7 \\
\hline TYK2 & 8.4 & 4.7 & 2.3 \\
\hline Tie2 & 2.8 & -0.5 & -1.2 \\
\hline Tpl2 & 18 & 16.3 & 23.5 \\
\hline TrkA & 15.2 & 13.1 & 6.2 \\
\hline TrkB & 6.2 & 4 & 7.2 \\
\hline WEE1 & 6.6 & 2.4 & 3.7 \\
\hline WNK2 & 3.8 & 4 & 6 \\
\hline YSK1 & -3 & -0.8 & 1.2 \\
\hline Yes & 7.9 & 4.9 & -3.9 \\
\hline ZAK & 2.6 & 4.5 & 8.4 \\
\hline ZAP-70 & 7.5 & -2.3 & -1.1 \\
\hline ZIPK & 1.3 & -0.1 & 4.2 \\
\hline eEF-2K & 5.5 & 2.4 & 3.9 \\
\hline mTOR & 3.7 & 1.3 & 4.1 \\
\hline p38_alpha & -1.7 & 4.5 & 4.9 \\
\hline p38_beta & 11.3 & 12.2 & 1 \\
\hline p38_delta & 12.1 & 8.2 & 6.3 \\
\hline p38_gamma & 3.9 & 8.2 & 9.3 \\
\hline p70S6K & 1.1 & 4.4 & 3.3 \\
\hline
\end{tabular}




\section{Synthetic procedures for $(\mathrm{R})-7$}
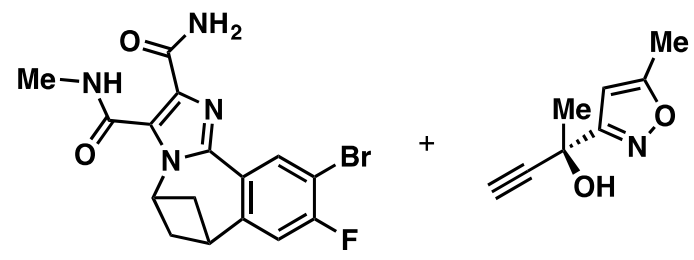

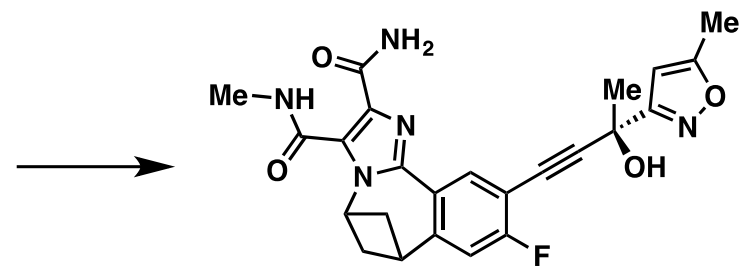

(R)-7

10-bromo-9-fluoro- $N^{3}$-methyl-6,7-dihydro-5 $H$-5,7-methanobenzo[c]imidazo[1,2a] azepine-2,3-dicarboxamide2 (0.32 mmol, $125 \mathrm{mg}, 1$ equiv) was suspended in triethylamine $(0.95 \mathrm{~mL})$ and acetonitrile $(0.95 \mathrm{~mL})$. The solution was degassed by sparging with nitrogen. CuI $(0.03 \mathrm{mmol}, 6 \mathrm{mg}, 0.1$ equiv $)$ and bis(triphenylphosphine)palladium(II) chloride $(0.03 \mathrm{mmol}, 22.3 \mathrm{mg}, 0.1$ equiv) were added as solids. A solution of (2R)-2-(5-methylisoxazol-3-yl)but-3-yn-2-ol (0.64 mmol, $96 \mathrm{mg}$, 2 equiv) in DMF (0.95 mL) was added dropwise. The reaction mixture was then heated at $80^{\circ} \mathrm{C}$ for $18 \mathrm{hr}$. Trituration with water, filtration and purification of the solid by reverse phase HPLC purification gave $19.5 \mathrm{mg}(13 \%)$ of (R)-9-fluoro-10-(3-hydroxy-3-(5-methylisoxazol-3-yl)but-1-yn-1-yl)- $N^{3}$-methyl-6,7dihydro-5H-5,7-methanobenzo[c]imidazo[1,2-a]azepine-2,3-dicarboxamide as a white solid.

\section{Analytical data for compound (R)-7:}

LCMS RT $=4.32 \mathrm{~min}, m / z=464.2[\mathrm{M}+\mathrm{H}]^{+}$

${ }^{1} \mathrm{H}$ NMR (400 MHz, DMSO- $d_{6}$ ) $\delta 10.62-10.56(\mathrm{~m}, 1 \mathrm{H}), 8.85(\mathrm{~d}, J=7.5 \mathrm{~Hz}, 1 \mathrm{H}$ ), 8.28 (s, 1H), $7.74(\mathrm{~s}, 1 \mathrm{H}), 7.32$ (d, J = $10.0 \mathrm{~Hz}, 1 \mathrm{H}), 6.58(\mathrm{~s}, 1 \mathrm{H}), 6.36(\mathrm{~s}, 1 \mathrm{H}), 6.12-6.08$ $(\mathrm{m}, 1 \mathrm{H}), 3.70-3.63(\mathrm{~m}, 1 \mathrm{H}), 3.16-3.07(\mathrm{~m}, 2 \mathrm{H}), 2.78(\mathrm{~d}, J=4.6 \mathrm{~Hz}, 3 \mathrm{H}), 2.41(\mathrm{~s}, 3 \mathrm{H})$, $1.83(\mathrm{~s}, 3 \mathrm{H}), 1.72-1.64(\mathrm{~m}, 2 \mathrm{H})$.

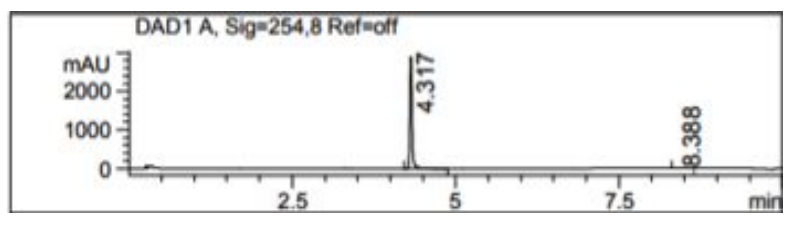




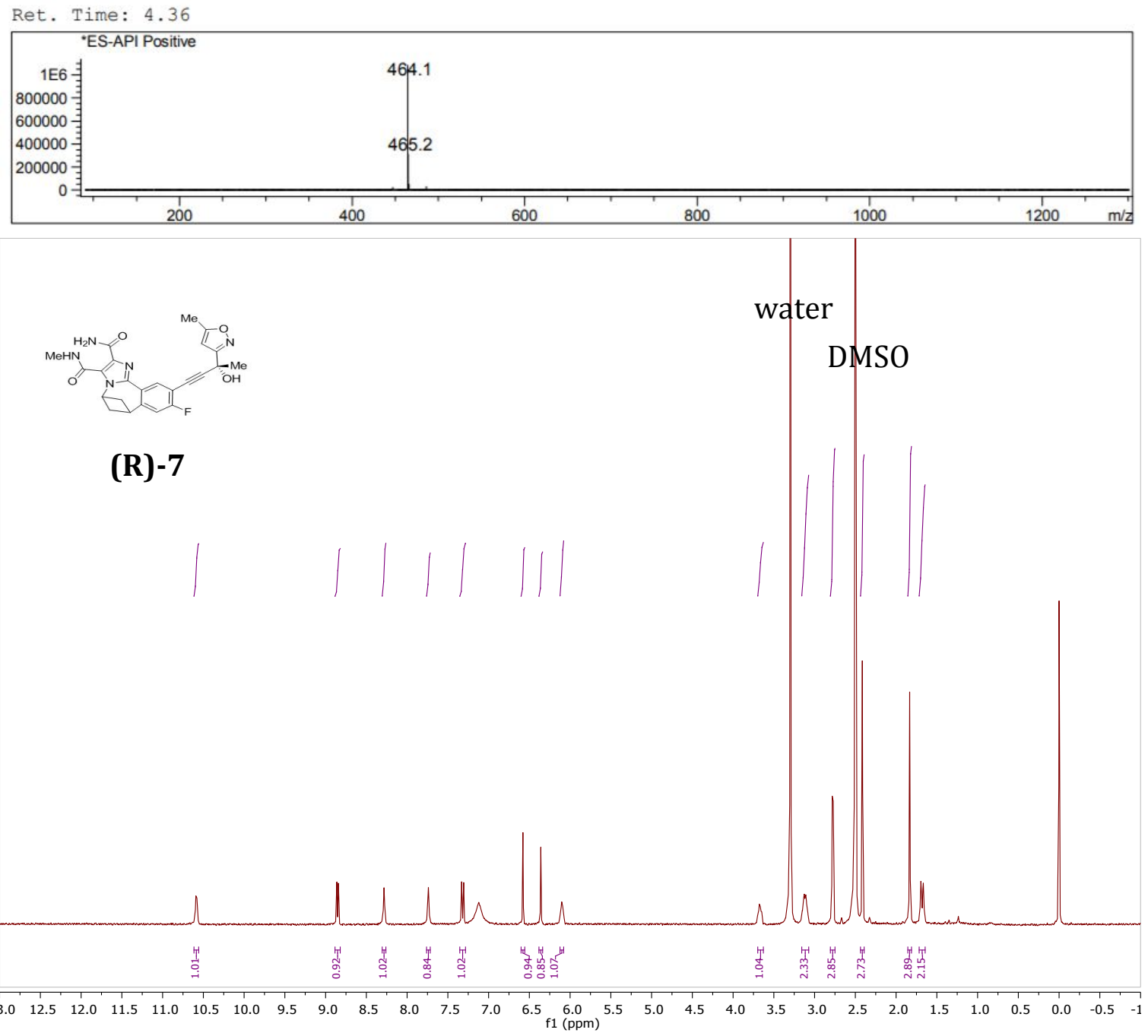

\section{Synthetic procedures for 10:}
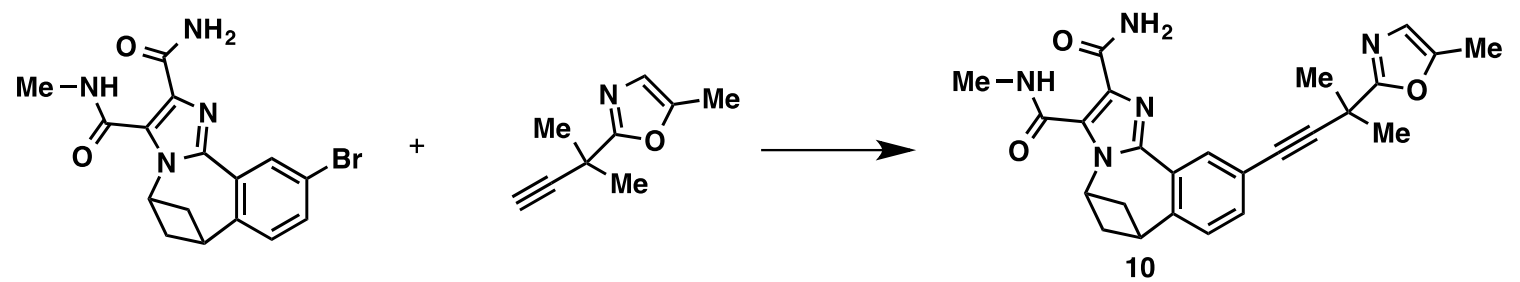

A 25-mL round-bottom flask was purged with nitrogen and charged with 10-bromo$N^{3}$-methyl-6,7-dihydro-5H-5,7-methanobenzo[c]imidazo[1,2-a]azepine-2,3- 
dicarboxamide ${ }^{2}$ (100 mg, $0.27 \mathrm{mmol}, 1.00$ equiv), degassed DMSO (5 mL), degassed triethylamine (5 mL), 5-methyl-2-(2-methylbut-3-yn-2-yl)oxazole (54 mg, 0.36 mmol, 1.50 equiv), $\mathrm{PdCl}_{2}\left(\mathrm{PPh}_{3}\right)_{2}(18 \mathrm{mg}, 0.03 \mathrm{mmol}, 0.10$ equiv). The reaction mixture was heated at $90^{\circ} \mathrm{C}$ for $3 \mathrm{hr}$. The mixture was cooled to room temperature and diluted with $30 \mathrm{~mL}$ of saturated $\mathrm{NH}_{4} \mathrm{Cl}$ (aq). The resulting solution was extracted with ethyl acetate and the organics washed with water and brine. The organics were dried over anhydrous sodium sulfate and concentrated under vacuum. The crude product was purified by Prep-HPLC with the following conditions: Column, X Bridge C18, 19*150 mm, 5 um; Mobile Phase A: Water $/ 0.05 \%$ $\mathrm{NH}_{4} \mathrm{HCO}_{3}$, Mobile Phase B: ACN; Flow rate: $30 \mathrm{~mL} / \mathrm{min}$; Detector, $254 \mathrm{~nm}$. This resulted in $63.6 \mathrm{mg}$ (54\%) of $N^{3}$-methyl-10-(3-methyl-3-(5-methyloxazol-2-yl)but1-yn-1-yl)-6,7-dihydro-5H-5,7-methanobenzo[c]imidazo[1,2-a]azepine-2,3-

dicarboxamide (compound 10) as a off-white solid.

\section{Analytical data for compound 10:}

LCMS: RT $=2.02 \mathrm{~min}, m / z=444.20[\mathrm{M}+\mathrm{H}]^{+}$

\section{Chromatogram}

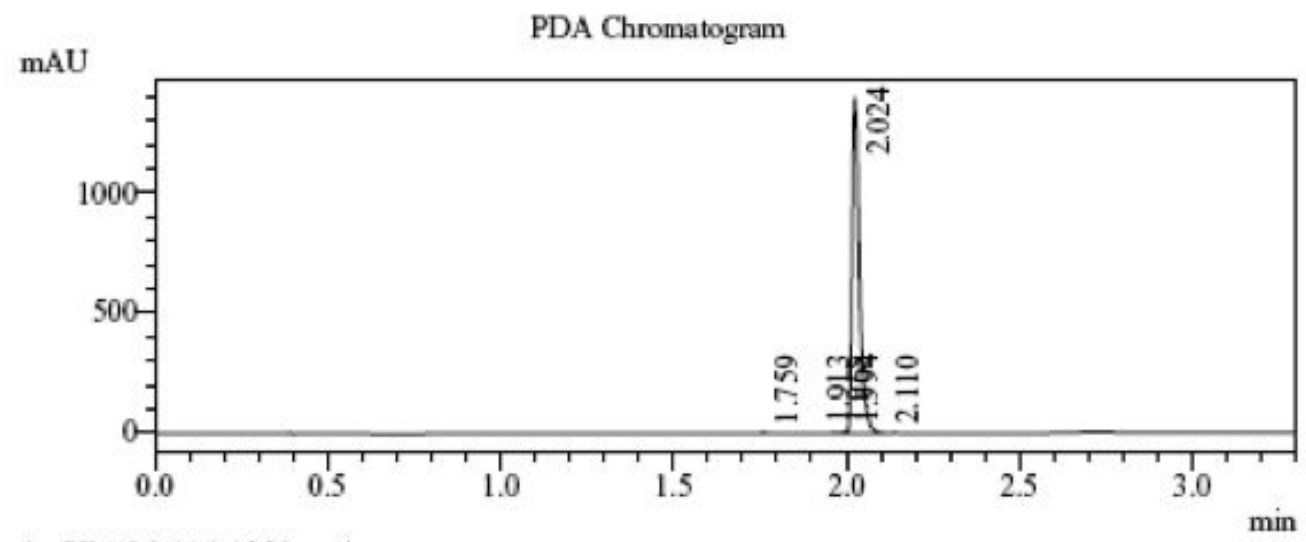

1 PDA Multi $1 / 254 \mathrm{~nm} 4 \mathrm{~nm}$ 


\section{MS Spectrum Graph}

MS Spectrum Graph

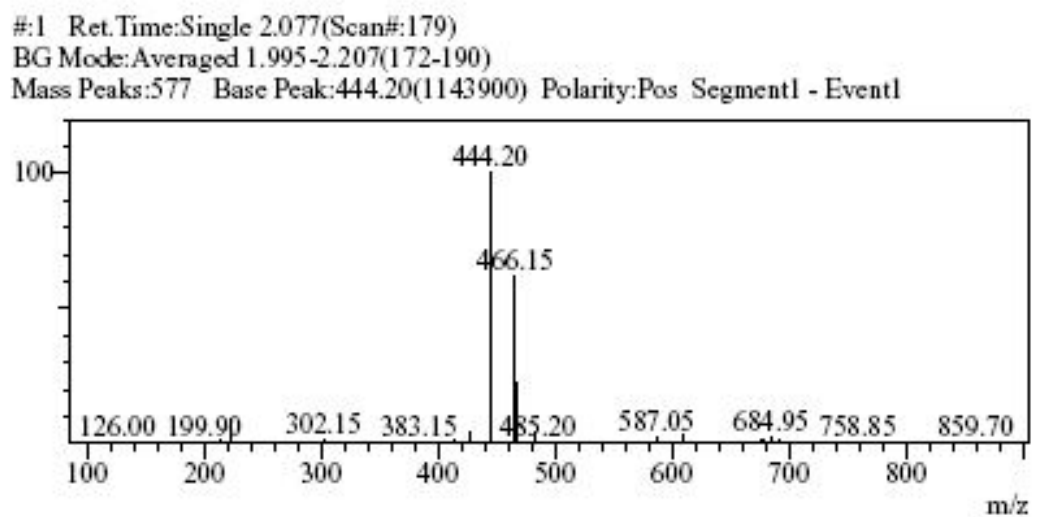

${ }^{1} \mathrm{H}-\mathrm{NMR}\left(300 \mathrm{MHz}\right.$, DMSO-d $\left.\mathrm{d}_{6}, p p m\right): \delta 10.56(\mathrm{~d}, J=4.5 \mathrm{~Hz}, 1 \mathrm{H}), 8.77(\mathrm{~d}, J=1.5 \mathrm{~Hz}, 1 \mathrm{H})$, 8.27(s, 1H), $7.75(\mathrm{~s}, 1 \mathrm{H}), 7.34-7.27(\mathrm{~m}, 2 \mathrm{H}), 6.79(\mathrm{~d}, J=1.2 \mathrm{~Hz}, 1 \mathrm{H}), 6.06-6.05(\mathrm{~m}, 1 \mathrm{H})$, 3.67-3.56 (m, $1 \mathrm{H}), 3.17-3.09(\mathrm{~m}, 2 \mathrm{H}), 2.77(\mathrm{~d}, J=4.5 \mathrm{~Hz}, 3 \mathrm{H}), 2.32-2.26(\mathrm{~m}, 3 \mathrm{H})$, $1.70-1.51(\mathrm{~m}, 8 \mathrm{H})$. 


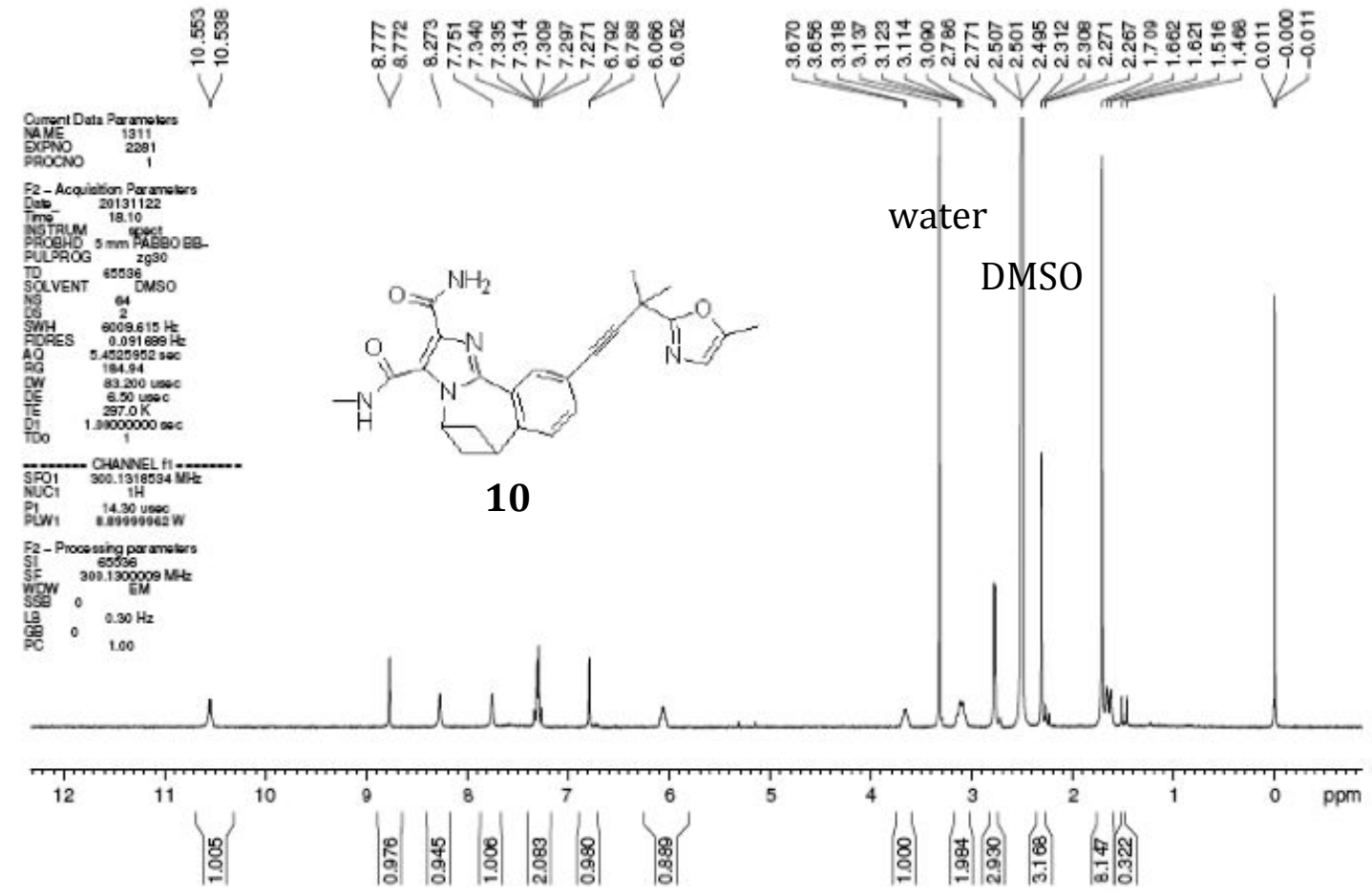

\section{Synthetic procedures for 11:}
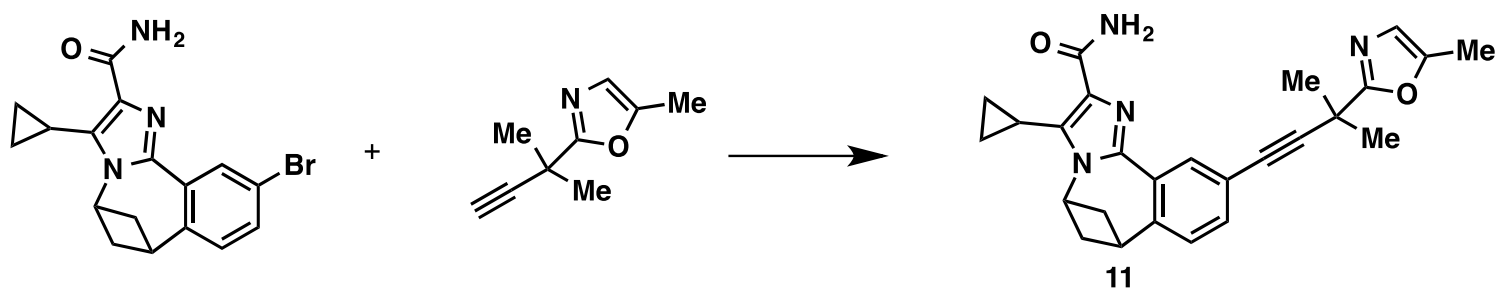

A 25-mL round-bottom flask was purged with nitrogen and charged with 10-bromo3-cyclopropyl-6,7-dihydro-5H-5,7-methanobenzo[c]imidazo[1,2-a]azepine-2carboxamide2 (100 mg, $0.28 \mathrm{mmol}, 1.00$ equiv), degassed DMSO (5 mL), degassed triethylamine (5 mL), 5-methyl-2-(2-methylbut-3-yn-2-yl)oxazole (55 mg, 0.36866 mmol, 1.50 equiv), $\mathrm{PdCl}_{2}\left(\mathrm{PPh}_{3}\right)_{2}(20 \mathrm{mg}, 0.03 \mathrm{mmol}, 0.10$ equiv). The resulting solution was heated at $90^{\circ} \mathrm{C}$ for $2 \mathrm{hr}$. The reaction mixture was cooled to room 
temperature, diluted with $30 \mathrm{~mL}$ of $\mathrm{NH}_{4} \mathrm{Cl}$ (aq) an the resulting solution was extracted with $2 \times 50 \mathrm{~mL}$ of ethyl acetate. The combined organics were washed with water and brine, dried over anhydrous sodium sulfate and concentrated under vacuum. The crude product was purified by Prep-HPLC with the following conditions: Column, X Bridge C18, 19*150 mm, 5 um; Mobile Phase A: Water $/ 0.05 \%$ NH4HCO3, Mobile Phase B: ACN; Flow rate: $30 \mathrm{~mL} / \mathrm{min}$; Detector, $254 \mathrm{~nm}$. This resulted in $29.1 \mathrm{mg}$ (24\%) of 3-cyclopropyl-10-(3-methyl-3-(5-methyloxazol-2yl)but-1-yn-1-yl)-6,7-dihydro-5H-5,7-methanobenzo[c]imidazo[1,2-a]azepine-2carboxamide (compound 11) as a white solid.

\section{Analytical data for compound 11:}

LCMS: $\mathrm{RT}=1.92 \mathrm{~min}, m / z=427.15[\mathrm{M}+\mathrm{H}]^{+}$

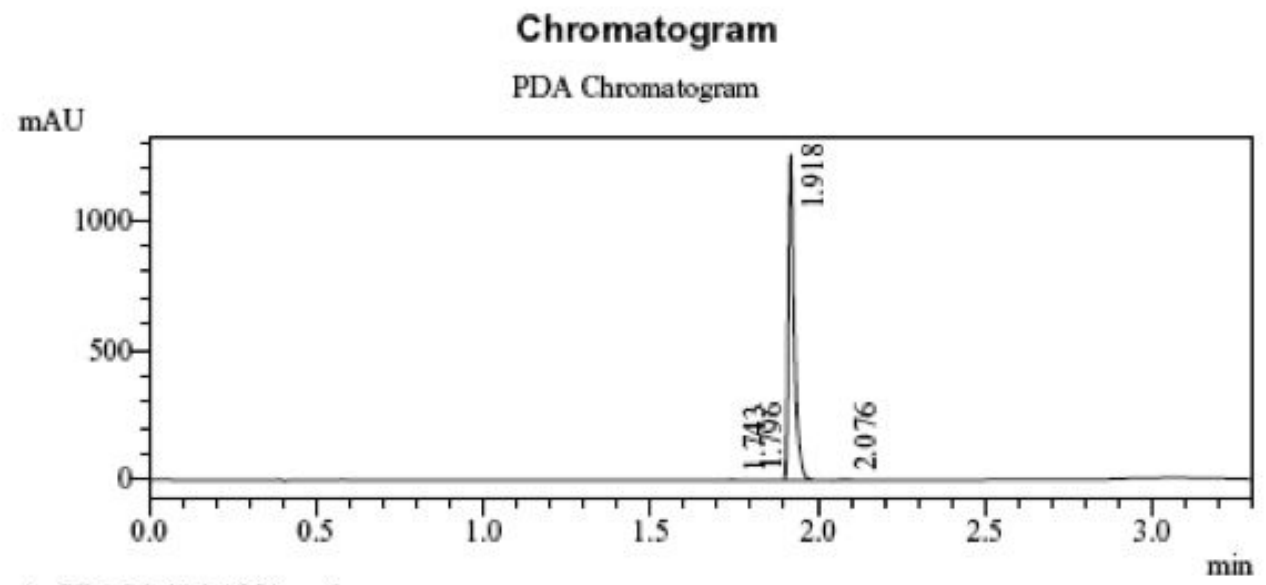

1 PDA Multi $1 / 254 \mathrm{~nm} 4 \mathrm{~nm}$ 
MS Spectrum Graph

MS Spectrum Graph

\#:1 Ret. Time:Single 1.972(Scan\#:170)

BG Mode:Averaged 1.913-2.059(165-177)

Mass Peaks:495 Base Peak:427.15(613138) Polarity:Pos Segmentl - Eventl

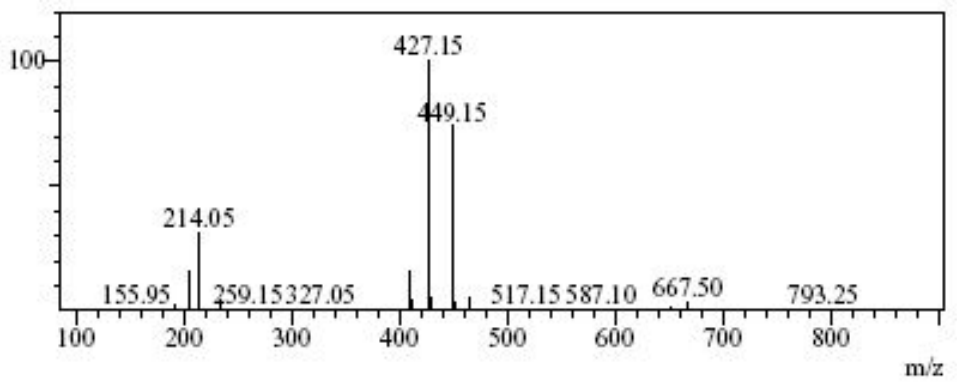

${ }^{1} \mathrm{H}-\mathrm{NMR}\left(300 \mathrm{MHz}\right.$, DMSO-d $\left.\mathrm{d}_{6}, p p m\right): \delta 8.61(\mathrm{~s}, 1 \mathrm{H}), 7.47(\mathrm{~s}, 1 \mathrm{H}), 7.23(\mathrm{~d}, J=0.9 \mathrm{~Hz}$, $2 \mathrm{H}), 6.96(\mathrm{~s}, 1 \mathrm{H}), 6.78(\mathrm{~d}, J=1.2 \mathrm{~Hz}, 1 \mathrm{H}), 5.30-5.24(\mathrm{~m}, 1 \mathrm{H}), 3.67-3.56(\mathrm{~m}, 1 \mathrm{H}), 3.13-$ $3.11(\mathrm{~m}, 2 \mathrm{H}), 2.30(\mathrm{~d}, J=1.2 \mathrm{~Hz}, 3 \mathrm{H}), 1.78-1.64(\mathrm{~m}, 9 \mathrm{H}), 1.03-0.96(\mathrm{~m}, 2 \mathrm{H}), 0.72-0.61$ $(\mathrm{m}, 2 \mathrm{H})$.
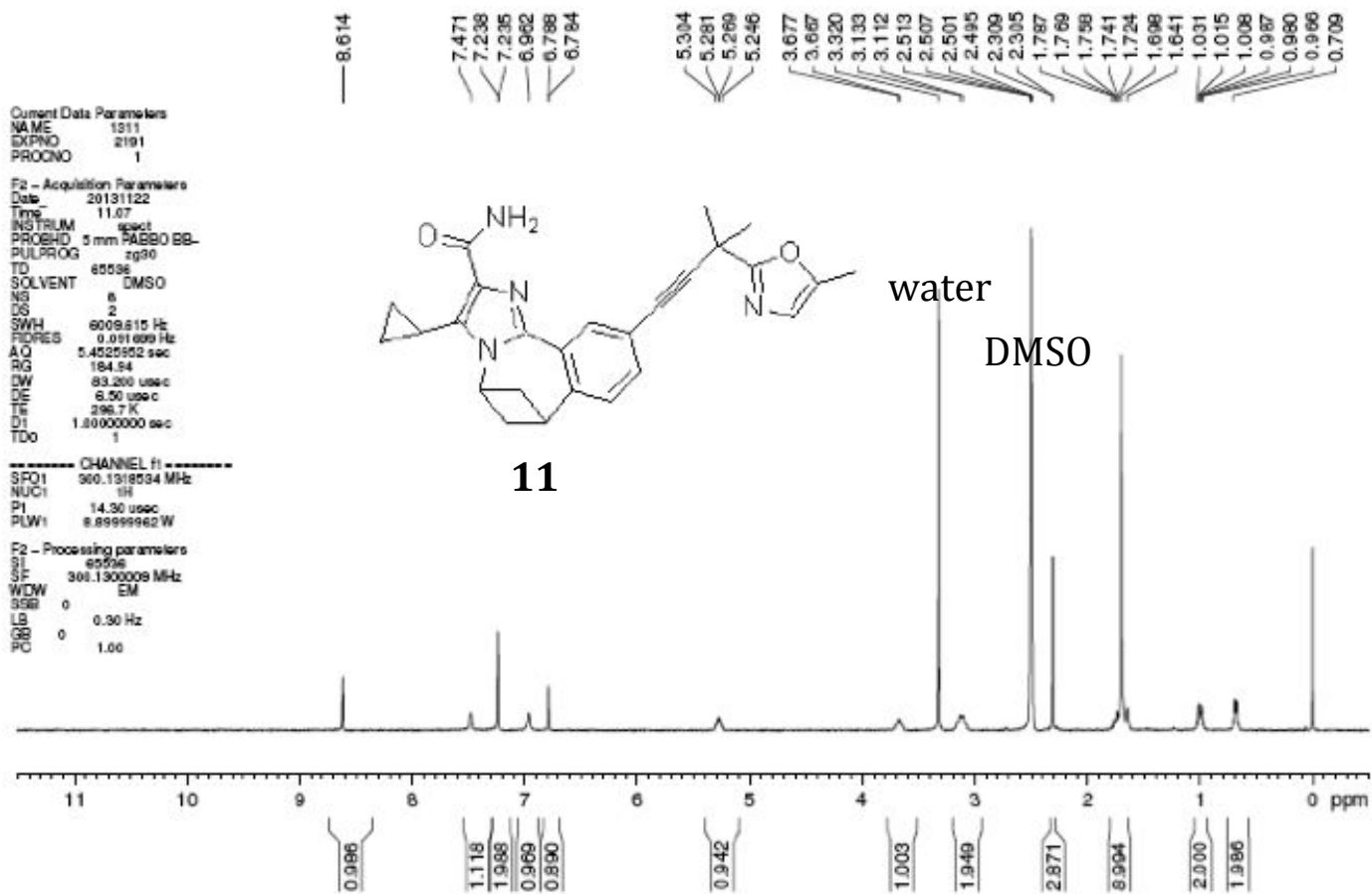


\section{Synthetic procedures for (S)-12}
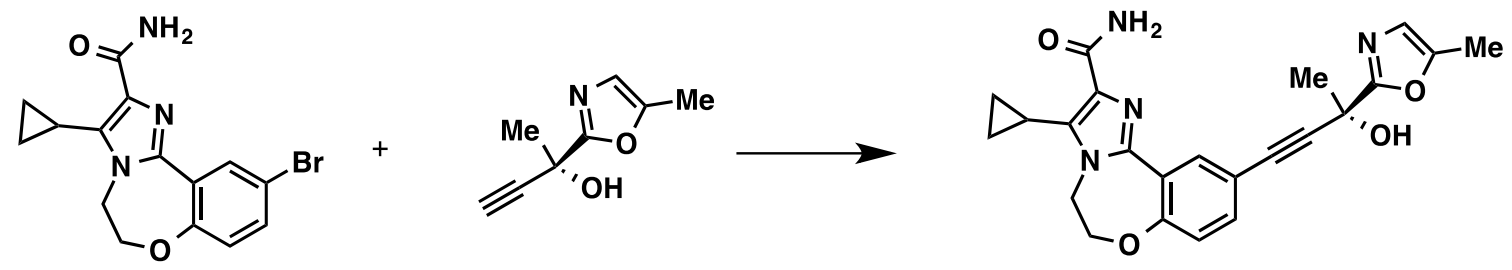

(S)-12

A 25-mL round-bottom under nitrogen was charged with 10-bromo-3-cyclopropyl5,6-dihydrobenzo[f]imidazo[1,2- $d][1,4]$ oxazepine-2-carboxamide2 (100 mg, 0.29 mmol, 1.00 equiv), degassed triethylamine (5 mL), degassed DMSO (5 mL), $(S)$-2-(5methyloxazol-2-yl)but-3-yn-2-ol (65 mg, $0.43 \mathrm{mmol}, 1.50$ equiv), and $\mathrm{PdCl}_{2}\left(\mathrm{PPh}_{3}\right)_{2}$ (20 mg, $0.03 \mathrm{mmol}, 0.10$ equiv). The resulting solution was heated at $90^{\circ} \mathrm{C}$ for $2 \mathrm{hr}$. The reaction mixture was cooled to room temperature, and diluted with $20 \mathrm{~mL}$ of $\mathrm{NH}_{4} \mathrm{Cl}_{(\mathrm{aq})}$ and extracted with $2 \times 50 \mathrm{~mL}$ of ethyl acetate. The organic layers were combined, washed with water and brine, dried over anhydrous sodium sulfate and concentrated under vacuum. The crude product was purified by Prep-HPLC with the following conditions: Column, X Bridge C18, 19*150 mm, 5 um; Mobile Phase A: Water/0.05\% NH4HCO3, Mobile Phase B: ACN; Flow rate: $30 \mathrm{~mL} / \mathrm{min}$; Detector, $254 \mathrm{~nm}$. This resulted in $22 \mathrm{mg}(18 \%)$ of $(S)$-3-cyclopropyl-10-(3-hydroxy-3-(5methyloxazol-2-yl)but-1-yn-1-yl)-5,6-dihydrobenzo[f]imidazo[1,2d] [1,4] oxazepine-2-carboxamide (compound (S)-12) as a off-white solid.

\section{Analytical data for compound (S)-12:}

LCMS RT =3.03 $\min , m / z=419.05[\mathrm{M}+\mathrm{H}]^{+}$ 


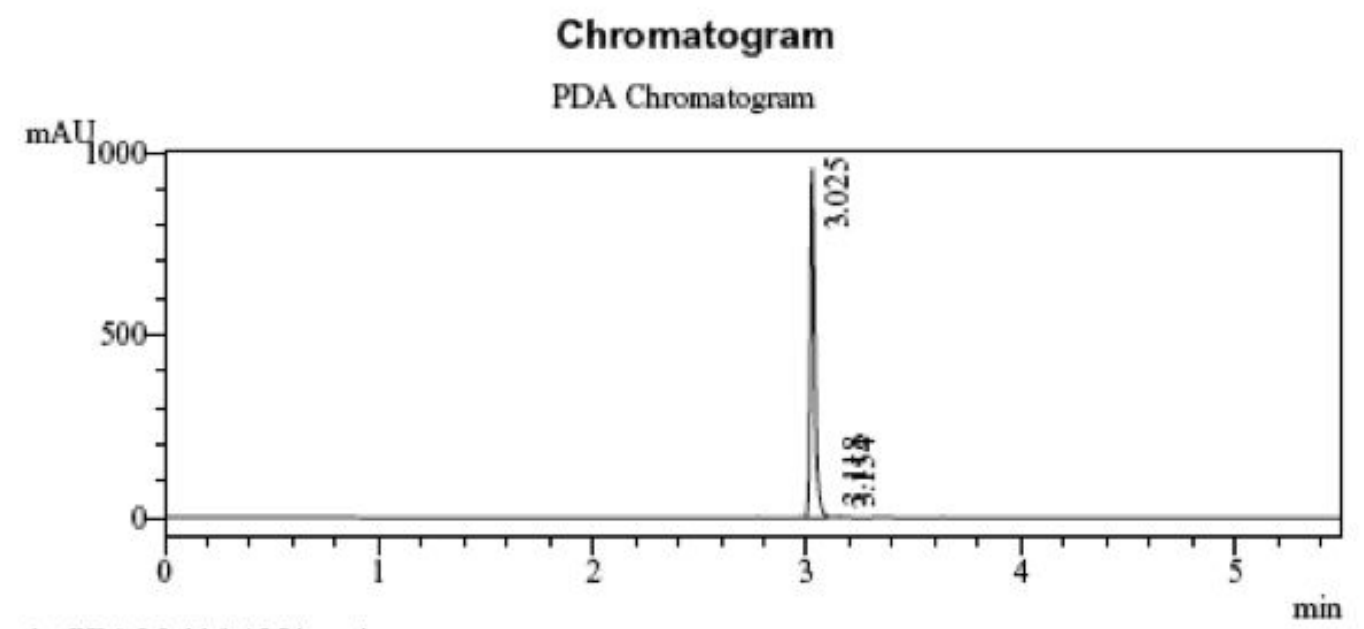

1 PDA Multi $1 / 254 \mathrm{~nm} 4 \mathrm{~nm}$

\section{MS Spectrum Graph}

$$
\text { MS Spectrum Graph }
$$

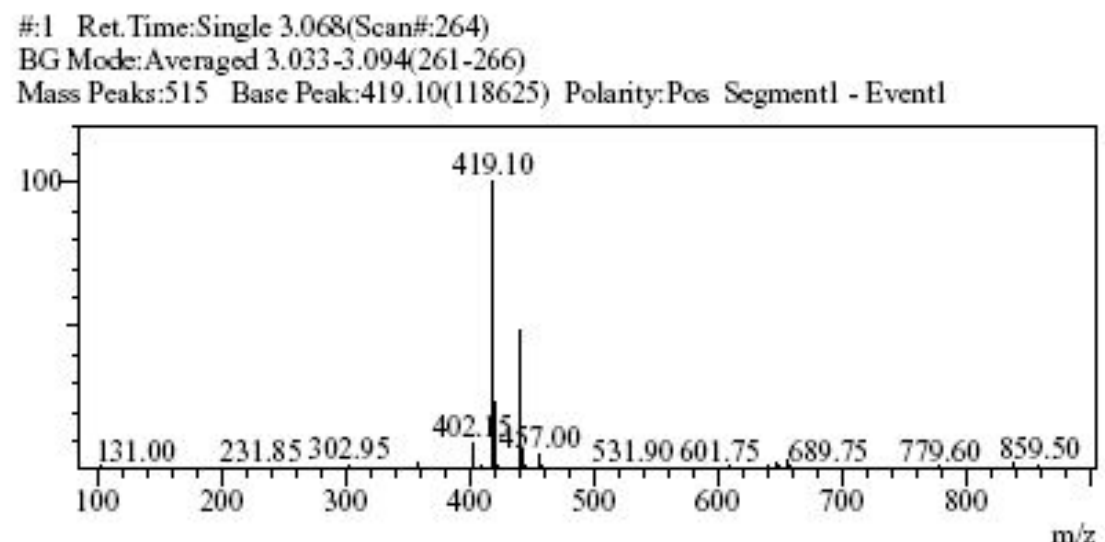

${ }^{1} \mathrm{H}-\mathrm{NMR}\left(300 \mathrm{MHz}, \mathrm{CD}_{3} \mathrm{OD}, p p m\right): \quad \delta 8.56(\mathrm{~d}, J=2.1 \mathrm{~Hz}, 1 \mathrm{H}), 7.45(\mathrm{~d}, J=8.7 \mathrm{~Hz}, 1 \mathrm{H})$, $7.22(\mathrm{~d}, J=8.7 \mathrm{~Hz}, 1 \mathrm{H}), 6.30$ (d, $J=0.9 \mathrm{~Hz}, 1 \mathrm{H}), 4.53(\mathrm{~s}, 4 \mathrm{H}), 2.44(\mathrm{~d}, J=0.6 \mathrm{~Hz}, 3 \mathrm{H}$ ), $1.87(\mathrm{~s}, 3 \mathrm{H}), 1.80-1.74(\mathrm{~m}, 1 \mathrm{H}), 1.15-1.09(\mathrm{~m}, 2 \mathrm{H}), 0.83-0.80(\mathrm{~m}, 2 \mathrm{H})$. 


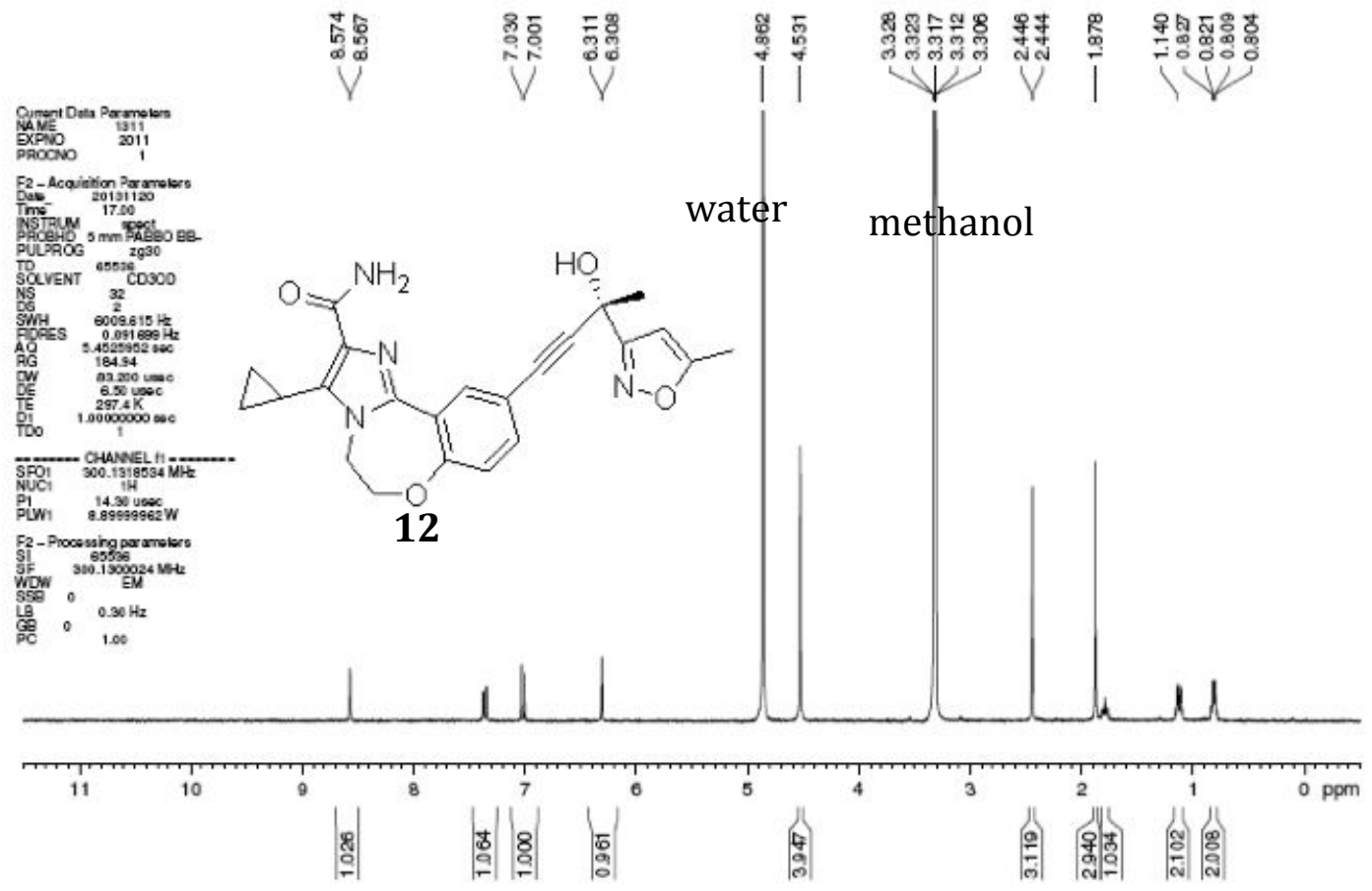




\section{Modeling software and procedures}

PKD1 model was build using the Homology Model workflow in the MOE v2012 modeling software. The modeling template was a NIK structure (4G3G) with an aminothiozole ligand bound. Sequence alignment between PKD1 and NIK was constrained to ensure conserved kinase features like gate keeper, catalytic lysine, hinge were aligned.

Models of ligands bound to a PKD1 homology model were build by overlaying ligand bound NIK crystal structures.

\section{References:}

${ }^{1}$ Crawford, T. D.; Ndubaku, C. O.; Chen, H.; Boggs, J. W.; Bravo, B. J.; DeLaTorre, K.; Giannetti, A. M.; Gould, S. E.; Harris, S. F.; Magnuson, S. R.; McNamara, E.; Murray, L. J.; Nonomiya, J.; Sambrone, A.; Schmidt, S.; Smyczek, T.; Stanley, M.; Vitorino, P.; Wang, L.; West, K.; Wu, P.; Ye, W. Discovery of Selective 4-Amino-Pyridopyrimidine Inhibitors of MAP4K4 Using Fragment-Based Lead Identification and Optimization. J. Med. Chem. 2014, 57 (8), 3484-3493.

${ }^{2}$ Prepared as described in - Castanedo, G.; Feng, J.; Montalbetti, C. A. G. N.; Staben, S. "Tricyclic compounds as NIK inhibitors and their preparation and use in the treatment of inflammatory diseases" WO 201320980.

${ }^{2}$ Crawford, T. D.; Ndubaku, C. O.; Chen, H.; Boggs, J. W.; Bravo, B. J.; DeLaTorre, K.; Giannetti, A. M.; Gould, S. E.; Harris, S. F.; Magnuson, S. R.; McNamara, E.; Murray, L. J.; Nonomiya, J.; Sambrone, A.; Schmidt, S.; Smyczek, T.; Stanley, M.; Vitorino, P.; Wang, L.; West, K.; Wu, P.; Ye, W. J. Med. Chem. 2014, 57 (8), 3484-3493. 